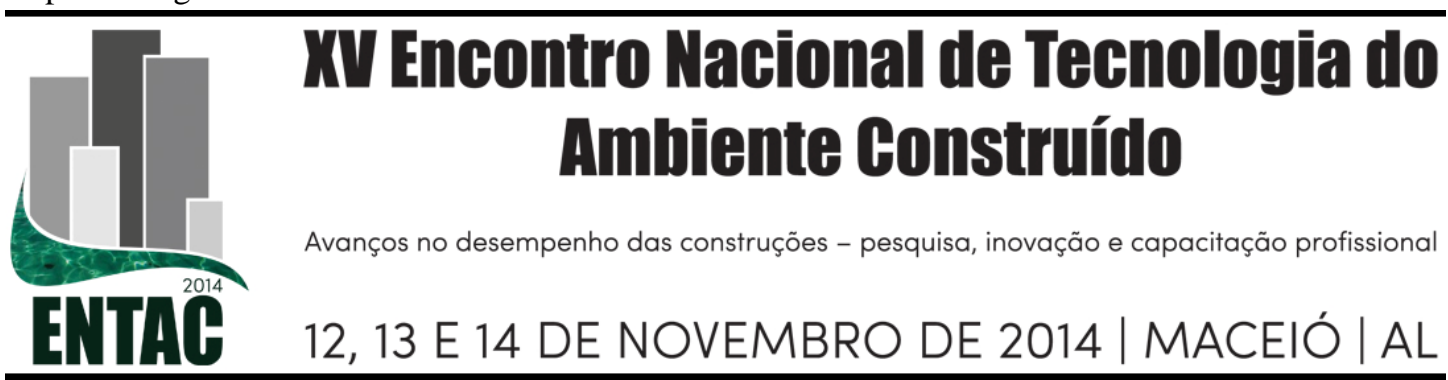

\title{
ANALISE DO IMPACTO DAS DECISÕES ARQUITETÔNICAS NA DEFINIÇÃO DA POTÊNCIA INSTALADA DO SISTEMA DE CONDICIONAMENTO DE AR ESTUDO DE CASO: PALÁCIO GUSTAVO CAPANEMA
}

\author{
SILVA, Sandra (1); SOUZA, Roberta (2) \\ (1) Engenheiro Mecânico, Mestre em Ambiente Construído e Patrimônio Sustentável - EA / UFMG, \\ sandra@protherm.com.br, (2) Dra, Professora da Escola de Arquitetura / UFMG, \\ robertavgs2@gmail.com
}

\begin{abstract}
RESUMO
Diversas decisões tomadas no desenvolvimento do projeto arquitetônico têm impacto no dimensionamento do sistema de Condicionamento de Ar a ser instalado, uma vez que influenciam diretamente na carga térmica a ser combatida. Entretanto, o impacto das decisões arquitetônicas no desempenho térmico da edificação e consequentemente no dimensionamento do sistema de Condicionamento de Ar, muitas vezes não é avaliado de forma conjunta e integrada, durante a elaboração do projeto. O presente estudo teve como objetivo apresentar a avaliação dos resultados dos cálculos da carga térmica para uma edificação específica, a saber, o Palácio Gustavo Capanema, localizado no Rio de Janeiro, tendo como base a utilização das medidas de proteção passiva adotadas no projeto original, bem como a possibilidade de redução da carga térmica, em função da variação do coeficiente de sombreamento do vidro. Além da avaliação da variação da carga térmica, foi estudado o impacto desta variação no dimensionamento da capacidade, na potência elétrica instalada e no consumo de energia do sistema de Condicionamento de Ar. O estudo apresenta parte dos resultados finais de dissertação de mestrado. O método adotado foi o estudo de caso de caráter exploratório. Com base nas simulações, realizadas adotando-se o software TRACE 700, foram elaboradas análises comparativas dos resultados da carga térmica, do investimento inicial, do consumo elétrico e do custo operacional da energia do sistema de Condicionamento de $\operatorname{Ar}$ (HVAC). Analisando os resultados obtidos, foi possível confirmar que as decisões arquitetônicas impactam de forma direta na carga térmica da edificação. Verificou-se também, através da análise dos resultados, que as variações da carga térmica obtidas apresentaram percentuais diferentes da variação do investimento inicial, da potência elétrica e do consumo, em função da modulação da capacidade dos equipamentos de HVAC existentes. Com o presente estudo fica evidenciada a importância do desenvolvimento do projeto de forma integrada, uma vez que se verifica que as decisões de cada disciplina, principalmente as arquitetônicas, impactam diretamente nas demais disciplinas e consequentemente no consumo de energia da edificação.
\end{abstract}

Palavras-chave: carga térmica, simulação, eficiência.

\begin{abstract}
Several decisions made during the development of the architectural design have direct implication on the results of the thermal loads considered in the dimensioning of air conditioning system, once they directly impact the thermal loads. This study presents a comparative evaluation of the results of the calculation of the thermal loads to a specific building, namely Gustavo Capanema Palace, located in Rio de Janeiro, based on the utilization of passive solar protections used in the original design, as well as, variations of the glass shading coefficient. Besides the evaluation of the variation of the thermal loads, the impact on the dimensioning of the capacity in the installed power and the electric power consumption of the air
\end{abstract}


conditioning system were studied. The simulations were carried out using the software TRACE 700. Based on the simulations, of the thermal loads was calculated and comparative analysis of the thermal loads, of the initial investment, of the electrical consumption and of the cost of the energy was carried out. It was verified that the architectural decisions impact directly on the thermal load of the building. It was also verified that the variation of the thermal load showed different values from the variation of the initial investment, electric power and consumption, due to of the modulation of the capacity of the commercial equipments. The present study brings light to the importance of developing an integrated design, once it is found that the decisions of each discipline, especially the architectural design, directly impact other disciplines.

Keywords: cooling load, simulation, energy efficiency

\section{INTRODUÇÃO}

A indústria da construção consome $50 \%$ dos recursos mundiais, o que faz dela uma das atividades menos sustentáveis do planeta. Não obstante, nossa vida cotidiana gira ao redor de uma grande quantidade de construções: vivemos em casas, utilizamos carros, trabalhamos em edifícios e utilizamos estabelecimentos comerciais. A civilização contemporânea depende das edificações para seu abrigo e existência e nosso planeta não suporta mais o aumento do consumo dos recursos naturais. Portanto, é evidente que algo tem que mudar e a arquitetura tem um papel importante a desempenhar nesta mudança (EDWARDS, 2008).

$\mathrm{O}$ ato de construir é por si só, um ato de natureza complexa. Centenas de processos precisam ser desenvolvidos antes que as fundações da edificação possam ser feitas, que as paredes possam ser erguidas, que o interior seja finalizado e que as pessoas possam ocupar o espaço projetado. Fica também muito claro que precisamos construir de forma mais sustentável: construir gastando menos energia, menos água, usando o mínimo possível os recursos finitos da natureza e ao máximo, os produtos reciclados (BOECKER, 2009).

Avaliar o desempenho energético das edificações é uma tarefa complexa que envolve grande quantidade de variáveis interdependentes e conceitos multidisciplinares. Através dos programas de simulação, pode-se avaliar o desempenho térmico e energético de edificações, para diferentes alternativas de projeto, sejam elas opções do desenho arquitetônico, dos componentes construtivos, dos sistemas de iluminação ou dos sistemas de condicionamento de ar. (MENDES, 2005).

\section{OBJETIVO}

O presente trabalho, por meio do estudo de caso do Palácio Gustavo Capanema, visa analisar a inter-relação entre as decisões arquitetônicas, o dimensionamento do sistema de HVAC e do sistema elétrico, bem como do consumo de energia e consequentemente do custo operacional de uma edificação.

\section{METODOLOGIA}

\subsection{Descrição do estudo de caso - Palácio Gustavo Capanema}

O prédio escolhido como estudo de caso foi o Palácio Gustavo Capanema (PGC), no Rio de Janeiro, atualmente em processo de restauro pelo IPHAN-RJ. O Palácio Gustavo Capanema é o símbolo mais impactante da arquitetura moderna no Brasil e a primeira aplicação em escala monumental das ideias de Le Coubusier, com parceria de arquitetos consagrados, entre eles, Lúcio Costa, Oscar Niemeyer, Affonso Eduardo Reidy, Jorge 
Machado Moreira, Carlos Leão e Ernany de Vasconcelos (IPHAN, 2012). Ele mostra claramente a força com que um prédio pode modelar o espaço em torno de si (MINDLIN, 2000).

$\mathrm{Na}$ elaboração do projeto para construção do PGC, na década de 30 e 40, foram adotadas noções de conforto ambiental, ventilação natural e cruzada, com utilização de diversos elementos arquitetônicos, específicos para o controle da incidência solar e da temperatura interna, o que caracteriza esta edificação como um modelo, adaptado a seu tempo, de construção sustentável (IPHAN, 2012).O acesso aos dados da edificação se deu através de consultoria, prestada por uma das autoras do presente artigo, para o estabelecimento de diretrizes básicas e conceituais para a contratação de projeto de condicionamento de ar, obedecendo às premissas de preservação estabelecidas no tombamento da edificação e às normas de proteção e conservação do IPHAN.
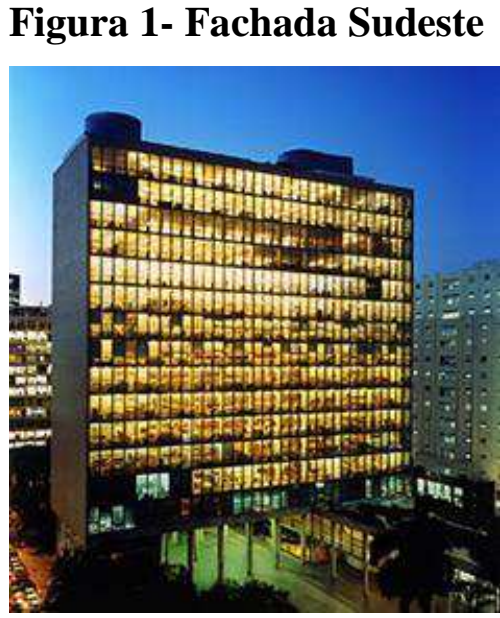

Fonte:

http://culture-e.com/noticias/588

Em função do interesse e da peculiaridade do Palácio Gustavo Capanema no contexto histórico da arquitetura moderna no Brasil e no mundo, o presente trabalho propôs ampliar o processo de consultoria, no sentido de fazer análises aprofundadas das possíveis decisões de projeto.

\subsubsection{Forma e orientação solar}

A forma da edificação e a orientação solar das fachadas Figura 2- Fachada Noroeste são aspectos arquitetônicos importantes em um projeto, pois irão impactar diretamente na quantidade de calor que entra no ambiente. No PGC, o prédio principal apresenta uma geometria retangular e possui 04 (quatro) fachadas. As fachadas Nordeste $\left(60^{\circ}\right)$ e Sudoeste $\left(240^{\circ}\right)$ são fachadas cegas. Já as fachadas Noroeste $\left(330^{\circ}\right)$ e Sudeste $\left(150^{\circ}\right)$ possuem vidro no seu fechamento, sendo que, no projeto original, foram previstas proteções solares para estas fachadas, a saber, brise soleil, na fachada Noroeste e persianas internas, na fachada Sudeste.

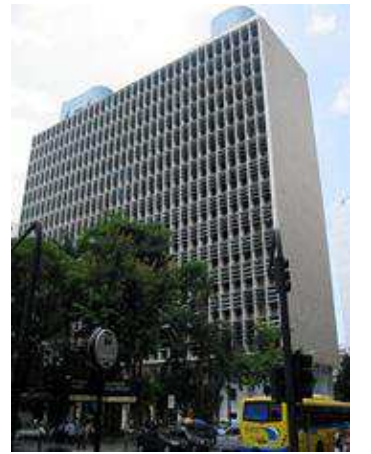

Fonte:

http://commons.wikimedia.org/wiki

\subsubsection{Recorte do Estudo de Caso}

O prédio do PGC é composto por dois blocos retangulares que se cruzam perpendicularmente. $\mathrm{O}$ bloco principal é um edifício vertical com 15 níveis $\left(2^{\circ}\right.$ ao $16^{\circ}$ pavimento), suspenso do solo por pilotis de $10 \mathrm{~m}$ de altura. Já o bloco anexo possui 2 níveis (Térreo e $1^{\circ}$ pavimento) é horizontal, passando por baixo do outro bloco, e abriga em seu teto um jardim concebido por Burle Marx. O presente estudo se ateve à avaliação do comportamento de parte do bloco principal da edificação, a saber, do $3^{\circ}$ ao $15^{\circ}$ pavimentos, de forma que a transmissão de calor do piso do $3^{\circ}$ pavimento e a do teto do $15^{\circ}$ pavimento, foram desconsideradas. As simulações foram feitas para um andar tipo, que foi subdividido em 03 (três) zonas térmicas, levando-se em conta a 
consideração, conforme definição do IPHAN, o fato de que o Hall de Elevadores não será climatizado, conforme apresentado na Figura 3.

\section{Figura 3 - Planta do Pavimento tipo}

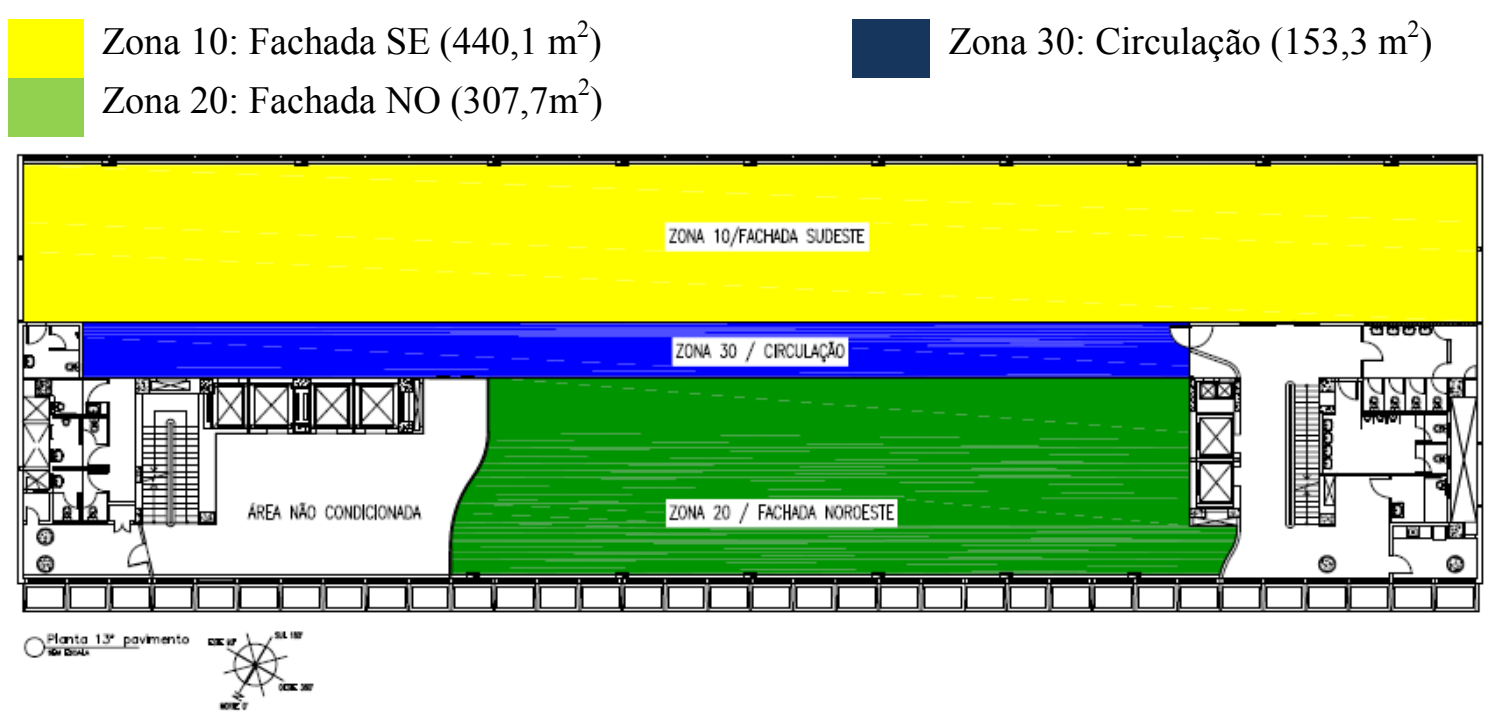

Fonte: SILVA, 2014

\subsection{Seleção do programa de simulação}

Para o desenvolvimento das simulações propostas neste estudo foi utilizado o programa de simulação TRACE 700 (Trane Air Conditioning Economics), que é um programa de cálculo de carga térmica e de análise econômico-energética, desenvolvido pelo grupo C.D.S. (Customer Direct Service Network), da empresa TRANE Company, programa este, acreditado no âmbito da ASHRAE 140-240, para de simulação de desempenho térmico e energético de edificações (TRANE 2010).

\subsection{Determinação das simulações}

Com o objetivo de avaliar o comportamento térmico da edificação, tendo em vista as alterações arquitetônicas propostas, foram realizadas 09 (nove) simulações, assim discriminadas:

Tabela 1 - Descrição das Simulações T1 à T9

\begin{tabular}{ll}
\hline \multicolumn{1}{c}{ Nomenclatura } & \multicolumn{1}{c}{ Descrição da simulação } \\
\hline T1_IPHAN_sBRsPE & Sem brise e sem persiana \\
\hline T2_IPHAN_BR0sPE & Brise na posição horizontal e sem persiana \\
\hline T3_IPHAN_BR45sPE & Brise $45^{\circ}$ e sem persiana \\
\hline T4_IPHAN_BR45PE0 & Brise $45^{\circ}$ e persiana posição horizontal \\
\hline T5_IPHAN_BR45PE45 * & Brise e persiana a 45 $^{\circ}$ ( situação atual ) \\
\hline T6_IPHANBR45PE45PR90 & Brise $45^{\circ}$, persiana na horizontal + película PR90 /3M \\
\hline T7_IPHAN_BR45PE45PR60 & Brise $45^{\circ}$,persiana na horizontal + película PR60/ 3M \\
\hline T8_IPHAN_BR45PE45VDHN & $\begin{array}{l}\text { Brise } 45^{\circ}, \text { persiana na horizontal+Vidro Habitat Neutro } \\
\text { Azul }(4 \mathrm{~mm})\end{array}$ \\
\hline T9_IPHAN_BR45PE45VDCL & $\begin{array}{l}\text { Brise a } 45^{\circ} \text { e persiana na horizontal+Vidro Cool Lite } 136 \\
\text { ST }(4 \mathrm{~mm})\end{array}$ \\
\hline
\end{tabular}

* A simulação T5_IPHAN_BR45_PE45 foi utilizada como a referência base para as comparações apresentadas a seguir, uma vez que esta opção representa o prédio nas 
condições originais de projeto, a saber, simulação com brise soleil, na fachada Noroeste e persianas internas, na fachada Sudeste. As simulações T1 à T4 foram realizadas com o objetivo de avaliar o comportamento da edificação, caso as medidas passivas adotadas no projeto original, não tivessem sido realizadas. As simulações T6 à T9 foram realizadas com o objetivo de avaliar a possível redução da carga térmica, em função da melhora no coeficiente de sombreamento dos vidros. As simulações foram realizadas adotando o critério da operação convencional do sistema de HVAC, a saber, funcionamento diurno de forma contínua, modulando sua capacidade de modo a manter a temperatura interna prevista de $24^{\circ} \mathrm{C}$ de TBS, ao longo do ano.

\subsection{Parâmetros de cálculo}

Em uma simulação, o modelo precisa apresentar características que definam cada uma das trocas de calor que compõem a carga térmica, de modo que, para que o modelo fosse simulado, foram adotados os parâmetros a seguir.

\subsubsection{Características dos elementos translúcidos}

Foram adotadas as seguintes características térmicas para os fechamentos translúcidos:

Tabela 2 - Características térmicas dos fechamentos translúcidos

\begin{tabular}{|c|c|c|c|c|}
\hline \multirow{3}{*}{ Descrição } & \multicolumn{4}{|c|}{ Características vedação translúcida } \\
\hline & \multicolumn{2}{|c|}{ Fachada Sudeste } & \multicolumn{2}{|c|}{ Fachada Noroeste } \\
\hline & $\mathbf{U}^{*}\left(\mathrm{~W} / \mathrm{m}^{2} .{ }^{\circ} \mathrm{C}\right)$ & CS* & $\mathrm{U}^{*}\left(\mathrm{~W} / \mathrm{m}^{2} .{ }^{\circ} \mathrm{C}\right)$ & CS (*) \\
\hline T1 IPHAN sBR sPE & 5,91 & 0,95 & 5,91 & 0,95 \\
\hline T2_IPHAN_BR0_sPE & 5,91 & 0,95 & 5,91 & 0,95 \\
\hline T3_IPHAN_BR45_sPE & 5,91 & 0,95 & 5,91 & 0,95 \\
\hline T4_IPHAN_BR45_PE0 & 4,60 & 0,90 & 5,91 & 0,95 \\
\hline T5_IPHAN_BR45_PE45 & 4,60 & $\mathbf{0 , 7 5}$ & 5,91 & $\mathbf{0 , 9 5}$ \\
\hline T6_IPHAN_BR45_PE45PR90 & 4,60 & 0,57 & 5,91 & 0,69 \\
\hline T7_IPHAN_BR45_PE45PR60 & 4,60 & 0,41 & 5,91 & 0,51 \\
\hline T8_IPHAN_BR45_PE45VDHN & 5,70 & 0,48 & 5,70 & 0,64 \\
\hline T9_IPHAN_BR45_PE45VDCL & 5,51 & 0,37 & 5,51 & 0,49 \\
\hline T10_IPHAN_BR45_PE0VN & 4,60 & 0,90 & 5,91 & 0,95 \\
\hline T11_IPHAN_BR45_PE45VN & 4,60 & 0,75 & 5,91 & 0,95 \\
\hline
\end{tabular}

Obs.: (*) Foram apresentadas neste artigo apenas algumas das características térmicas adotadas na simulação, entretanto, é importante ressaltar que o software adota como metodologia para o cálculo da carga térmica o método CLTD / CLF (Cooling Load Temperature Difference / Cooling Load Temperature), com a utilização do método TFM (Transfer Function Method) para gerar os dados utilizados na metodologia, de modo que é possível exercer controle sobre aspectos de transmitância térmica, armazenamento e/ou resposta térmica das massas que compõem a edificação (TRANE, 2010).

Os coeficientes de sombreamento (CS) apresentados contemplam não só o material de vedação translúcido, mas todo o conjunto, a saber: vidro simples, vidro + persiana e vidro + película + persiana. Para o vidro aramado, existente na fachada Sudeste foram adotadas as seguintes características térmicas: $\mathrm{U}=5,91 \mathrm{~W} / \mathrm{m}^{2} .{ }^{\circ} \mathrm{C}$ e $\mathrm{CS}=0,56$.

\subsubsection{Características construtivas das paredes}

As paredes internas ( Partions) e externas (Wall) apresentam a transmitância térmica de $2,28 \mathrm{~W} / \mathrm{m}^{2} .{ }^{\circ} \mathrm{C}$ e $2,29 \mathrm{~W} / \mathrm{m}^{2} .{ }^{\circ} \mathrm{C}$, respectivamente, tendo como base as construções adotadas, a partir da biblioteca do TRACE 700. 
Figura 4 - Descrição dos componentes das paredes internas e externas
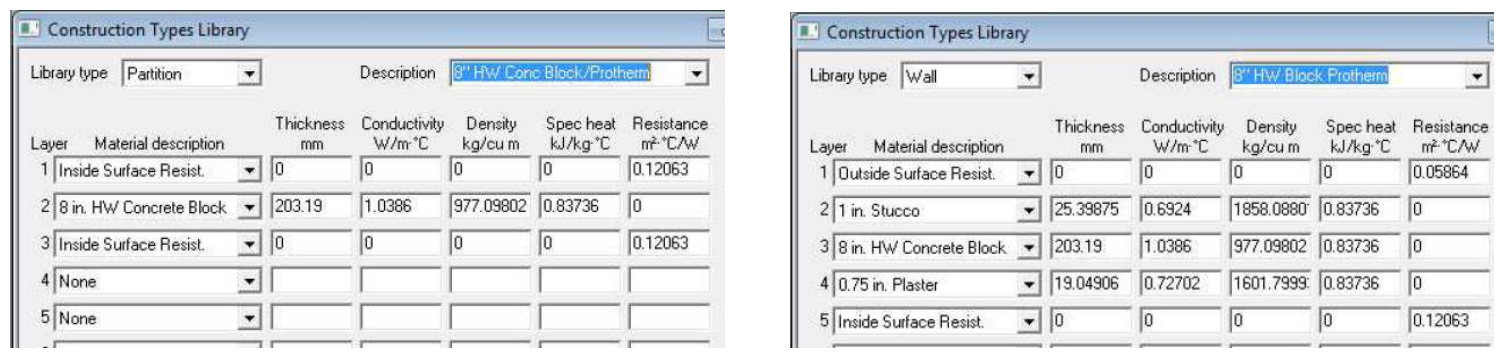

\subsubsection{Cargas internas}

- Ocupação dos espaços:

- Dissipação de calor / Pessoas:

$7 \mathrm{~m}^{2} /$ pessoa ( 99 pessoas/pavto )

Calor sensível $=>73,25 \mathrm{~kW} /$ pessoa

Calor latente $\quad \Rightarrow 58,6 \mathrm{~kW} /$ pessoa

- Dissipação Iluminação / Equipamentos:

$10 \mathrm{~W} / \mathrm{m}^{2}$ e $16 \mathrm{~W} / \mathrm{m}^{2}$

- Taxa de renovação de ar:

$27 \mathrm{~m}^{3} / \mathrm{h}$, conforme NBR 16401 .

\subsubsection{Condições Térmicas}

- Temperatura de Bulbo Seco (TBS):

\section{Internas}

$24{ }^{\circ} \mathrm{C}$

$50 \%$

\section{Externas}

Conforme arquivo

climático EPW

\subsubsection{Schedule de operação}

Uma vez que a ocupação dos espaços não se faz de forma imediata, para as simulações foi previsto o seguinte programa (Schedule) de operação:

Tabela 3 - Schedule de operação

\begin{tabular}{l|c|c|c}
\hline \multicolumn{1}{c|}{ Período } & Ocupação & Iluminação & Equipamentos \\
\hline Meia-noite - 7 a.m. & $0 \%$ & $0 \%$ & $0 \%$ \\
\hline 7 a.m. - 9 a.m. & $50 \%$ & $100 \%$ & $100 \%$ \\
\hline 9 a.m. - Meio-dia & $100 \%$ & $100 \%$ & $100 \%$ \\
\hline Meio-dia - 2 p.m. & $50 \%$ & $100 \%$ & $50 \%$ \\
\hline 2 p.m. - 6 p.m. & $100 \%$ & $100 \%$ & $100 \%$ \\
\hline 6 p.m. - 7 p.m. & $50 \%$ & $100 \%$ & $100 \%$ \\
\hline 7 p.m. - 11 p.m. & $0 \%$ & $100 \%$ & $0 \%$ \\
\hline 11 p.m - Meia-noite & $0 \%$ & $0 \%$ & $0 \%$ \\
\hline
\end{tabular}

\subsection{Equipamentos}

O sistema de condicionamento de ar definido para ser utilizado na edificação, conforme estudos elaborados junto com o IPHAN foi o sistema do tipo Multi Split "VRF". Foi então definida para cada simulação, a quantidade de unidades evaporadoras e o modelo da respectiva unidade condensadora a ser instalado no pavimento, adotando-se como referência os equipamentos da linha Multi V- III, da marca LG, conforme apresentado na Tabela 5 dos resultados. O COP das máquinas selecionadas foi sempre superior a $4,45 \mathrm{~W} / \mathrm{W}$. 


\section{RESULTADOS}

Com o objetivo de avaliar o impacto das decisões arquitetônicas no cálculo da carga térmica e no consumo de energia, em função do dimensionamento do sistema de condicionamento de ar, os resultados obtidos foram tratados de forma estatística, sendo elaboradas planilhas e gráficos com os resultados, de modo a possibilitar a análise comparativa dos valores obtidos.

\subsection{Comparativo da Carga Térmica}

A Tabela 4 apresenta a variação da carga térmica de pico simultânea, calculada para um pavimento tipo da edificação em estudo, para as simulações T1 à T9 .

\section{Tabela 4 - Comparação da carga térmica de pico calculada por pavimento}

\begin{tabular}{l|c|c}
\hline \multicolumn{1}{c|}{ Simulação } & $\begin{array}{c}\text { Carga Térmica } \\
\text { Total /Pavimento } \\
(\mathbf{~ k W ~})\end{array}$ & $\begin{array}{c}\text { Variação } \\
\text { Carga } \\
\text { Total }\end{array}$ \\
\hline T1_IPHAN_sBR_sPE & 147,0 & $12,5 \%$ \\
\hline T2_IPHAN_BR0_sPE & 138,9 & $6,4 \%$ \\
\hline T3_IPHAN_BR45_sPE & 138,8 & $6,3 \%$ \\
\hline T4_IPHAN_BR45_PE0 & 134,9 & $3,3 \%$ \\
\hline T5_IPHAN_BR45_PE45 & $\mathbf{1 3 0 , 6}$ & - \\
\hline T6_IPHAN_BR45_PE45_PR90 & 123,2 & $-5,7 \%$ \\
\hline T7_IPHAN_BR45_PE45_PR60 & 117,6 & $-9,9 \%$ \\
\hline T8_IPHAN_BR45_PE45_VDHN & 123,0 & $-5,8 \%$ \\
\hline T9_IPHAN_BR45_PE45_VDCL & 118,4 & $-9,3 \%$ \\
\hline
\end{tabular}

Comparando-se os resultados obtidos nas simulações $\mathrm{T} 1$ (sem brise e sem persianas), T2 (com brise horizontal e sem persianas), T3 (com brise angulado a $45^{\circ}$ e sem persianas), T4 (com brise angulado a $45^{\circ}$ e com persianas na horizontal) e T5 (com brise e persianas anguladas a $45^{\circ}$ ), foi possível avaliar, o impacto na carga térmica, das medidas passivas adotadas no projeto original, a saber, a instalação do brise, na fachada Noroeste e das persianas, na fachada Sudeste. Comparando os valores obtidos nas simulações T5 e T1, verificou-se que, caso o brise não houvesse sido instalado, a carga térmica total apresentaria um aumento de 12,5\%. Comparando os resultados obtidos na simulação T5 (com brise e persianas anguladas a $45^{\circ}$ ), com os resultados das simulações que propuseram a melhoria na eficiência térmica do vidro, a saber, a aplicação de película no vidro (simulações T6 e T7) e a troca do vidro existente por um vidro mais eficiente (simulações T7 e T8), foi possível avaliar que, no caso da aplicação da película no vidro, a redução da carga térmica global variou entre $-5,7 \%$ e $-9,9 \%$ (simulações T6 e T7), e no caso do vidro, tendo como base os vidros propostos, a variação ficou entre $-5,8 \%$ e $-9,3 \%$ (simulações T8 e T9).

\subsection{Arquitetura do Sistema de Condicionamento de Ar}

Os valores de investimento apresentados na Tabela 5 se referem exclusivamente ao custo dos equipamentos do sistema de HAVC e de sua instalação, conforme estimativa de mercado. Não foram incluídos no presente estudo, os custos que serão iguais para todas as opções, como por exemplo, o sistema de pressurização do ar externo e o sistema de automação, sendo que os valores apresentados se referem ao custo de apenas um pavimento. 
Tabela 5 - Resumo dos equipamentos, potência elétrica e investimento inicial do sistema de HVAC por pavimento / Simulações T1 à T9

\begin{tabular}{c|c|c|c|c|c|c}
\hline Simulação & $\begin{array}{c}\text { Modelo de } \\
\text { Referência } \\
\text { ( Marca LG) }\end{array}$ & $\begin{array}{c}\text { Capacidade } \\
\text { Térmica } \\
\text { Efetiva } \\
\text { ( kW })\end{array}$ & $\begin{array}{c}\text { Potência } \\
\text { elétrica } \\
\text { UC+ UE`s } \\
\text { ( kW) }\end{array}$ & $\begin{array}{c}\text { COP } \\
\text { ( W/W) }\end{array}$ & $\begin{array}{c}\text { Investi- } \\
\text { mento } \\
\text { Inicial } \\
\text { ( R\$) }\end{array}$ & $\begin{array}{c}\text { Varia- } \\
\text { ção }\end{array}$ \\
\hline T1 & ARUN 560 & 149,0 & 33,5 & 4,45 & 391.300 & $18,6 \%$ \\
\hline T2 & ARUN 540 & 143,6 & 31,6 & 4,54 & 290.895 & $8,0 \%$ \\
\hline T3 & ARUN 540 & 143,6 & 31,6 & 4,54 & 290.895 & $8,0 \%$ \\
\hline T4 & ARUN 520 & 138,3 & 29,9 & 4,63 & 279.110 & $3,6 \%$ \\
\hline T5 & ARUN 500 & $\mathbf{1 3 3 , 0}$ & $\mathbf{2 8 , 5}$ & $\mathbf{4 , 6 7}$ & $\mathbf{2 6 9 . 3 2 5}$ & - \\
\hline T6 & ARUN 480 & 127,7 & 27,3 & 4,68 & 268.675 & $-0,2 \%$ \\
\hline T7 & ARUN 460 & 122,4 & 26,8 & 4,57 & 254.490 & $-5,5 \%$ \\
\hline T8 & ARUN 480 & 127,7 & 27,3 & 4,68 & 259.390 & $-3,7 \%$ \\
\hline T9 & ARUN 460 & 122,4 & 26,8 & 4,57 & 259.390 & $-3,7 \%$ \\
\hline
\end{tabular}

Analisando os valores apresentados, verifica-se que a variação da carga térmica implica na mudança do modelo de condicionador adotado, o que tem como consequência a variação da potência elétrica, bem como do investimento inicial do sistema de HVAC.

\subsection{Comparativo do custo operacional}

A tarifa de energia para a edificação, conforme dados obtidos por meio das contas de energia, é um tipo de tarifa convencional que cobra por $\mathrm{kWh}$ consumido e um valor fixo pela demanda contratada, independente do valor consumido. Conforme conta de energia atual do prédio (junho / 2013), os valores cobrados pela Light para o PGC, com impostos inclusos e adotados nas simulações foram os seguintes: R\$ 0,1995781 / kWh e $\mathrm{R} \$ 95,64 \mathrm{R} \$ / \mathrm{kW}$ instalado .

Com base no perfil operacional e nos equipamentos propostos, foram obtidos os resultados mensais para o consumo elétrico dos equipamentos do sistema de HVAC, por pavimento, conforme apresentado na Figura 5.

Figura 5 - Comparação do consumo mensal por pavimento do sistema de HVAC - Simulações T1 à T9

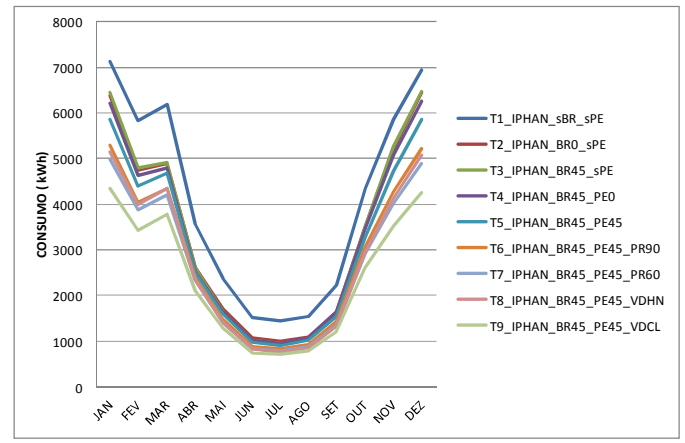

Fonte: SILVA, 2014

\section{Figura 6 - Comparação do consumo anual por pavimento do sistema de HVAC Simulações T1 à T9}

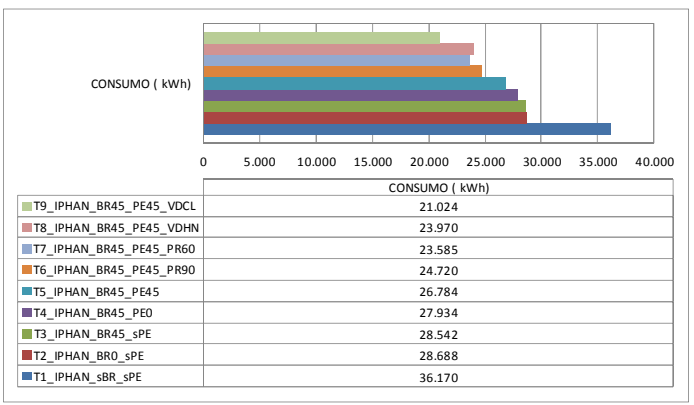

Fonte: SILVA, 2014

A Figura 6 apresenta, graficamente, o comparativo anual do consumo de energia, dos equipamentos do sistema de HVAC por pavimento, para as simulações T1 à T9.

A demanda a ser contratada na Concessionária depende da potência elétrica máxima a ser instalada, que por sua vez é função da capacidade de refrigeração do sistema e das 
demais cargas elétricas da edificação. O valor da demanda, apresentado na Tabela 6, se refere apenas à potência elétrica do sistema de HVAC.

\subsubsection{Custo da energia}

Com base nos custos de energia, apresentados no item 4.3, bem como nos valores de consumo e da demanda obtidos através das simulações, foram obtidos os seguintes resultados para os custos da energia, consumida pelo sistema de HVAC da edificação.

Tabela 2 - Resumo do custo anual da energia / HVAC - Simulações T1 à T9

\begin{tabular}{c|c|c|c|c|c|c|c}
\hline \multirow{2}{*}{ Simulação } & \multicolumn{2}{|c|}{ Consumo Total } & \multicolumn{3}{c|}{ Demanda Total } & \multicolumn{2}{c}{ Custo Total ANUAL } \\
\cline { 2 - 8 } & $\begin{array}{c}\text { Custo } \\
\text { ANUAL } \\
(\mathbf{R} \$)\end{array}$ & $\mathbf{\%}$ & $\begin{array}{c}\text { Potência } \\
(*) \\
(\mathbf{K w})\end{array}$ & $\begin{array}{c}\text { Custo } \\
\text { ANUAL } \\
(\mathbf{R} \$ \mathbf{~}\end{array}$ & $\mathbf{\%}$ & $\mathbf{R \$}$ & \% \\
\hline $\mathrm{T} 1$ & 127.045 & 31,0 & 465 & 533.671 & 19,2 & 660.716 & 21,3 \\
\hline $\mathrm{T} 2$ & 104.702 & 8,0 & 435 & 499.241 & 11,5 & 603.943 & 10,9 \\
\hline $\mathrm{T} 3$ & 104.477 & 7,8 & 435 & 499.241 & 11,5 & 603.718 & 10,9 \\
\hline $\mathrm{T} 4$ & 101.863 & 5,1 & 410 & 470.549 & 5,1 & 572.412 & 5,1 \\
\hline T5 & $\mathbf{9 6 . 9 5 8}$ & - & $\mathbf{3 9 0}$ & $\mathbf{4 4 7 . 5 9 5}$ & - & $\mathbf{5 4 4 . 5 5 4}$ & - \\
\hline $\mathrm{T} 6$ & 88.778 & $-8,4$ & 380 & 436.118 & $-2,6$ & 524.896 & $-3,6$ \\
\hline $\mathrm{T} 7$ & 84.289 & $-13,1$ & 370 & 424.642 & $-5,1$ & 508.931 & $-6,5$ \\
\hline $\mathrm{T} 8$ & 86.081 & $-11,2$ & 380 & 436.118 & $-2,6$ & 522.200 & $-4,1$ \\
\hline $\mathrm{T} 9$ & 74.670 & $-23,0$ & 370 & 424.642 & $-5,1$ & 499.312 & $-8,3$ \\
\hline
\end{tabular}

(*) Demanda prevista para o sistema de HVAC

Apesar de o consumo ter apresentado uma variação de $31 \%$ para a simulação T1 e - 23\% para a simulação T9, tendo como referência a simulação T5, o custo global da energia apresentou uma variação menor, a saber, $21,3 \%$ e $-8,3 \%$, respectivamente para as simulações T1 e T9, conforme apresentado na Tabela 6 . A variação do custo da demanda ficou entre $19,2 \%$, para a simulação T1 e $-5,1 \%$, para a simulação T9, enquanto que a variação no consumo, conforme citado anteriormente ficou entre $-21,3 \%$ e - 8,3\%, respectivamente para as simulações T1 e T9.

A variação diferenciada, em termos percentuais, entre o consumo $(\mathrm{kWh})$ e o custo da energia (R\$) se deve ao fato de que, o custo total da energia é composto por 02 (duas) parcelas distintas, a saber, pelo valor do consumo, acrescido do valor da demanda. Com o tipo de contrato de fornecimento de energia existente, independente da redução no consumo, a parcela da demanda é cobrada de forma integral.

\section{CONCLUSÕES}

Foram realizadas 09 (nove) simulações paramétricas da edificação, sendo que em cada uma delas foram simuladas $8760 \mathrm{~h}$. Nos modelos propostos foram variados os parâmetros referentes aos fechamentos translúcidos da edificação, sendo analisados os seguintes itens: (a) instalação e angulação do brise soleil, (b) instalação e angulação das persianas, (c) aplicação de película no vidro existente e (d) troca do vidro existente por um vidro termicamente mais eficiente.

Os resultados das simulações permitiram a análise do impacto conjunto das medidas propostas, bem como avaliar o impacto isolado da alteração destes parâmetros, além de mostrarem a importância da utilização de elementos de proteção solar e de vidros de alta eficiência térmica, objetivando a redução da carga térmica e consequentemente 
melhorando o desempenho energético da edificação. $O$ desempenho energético das simulações mostrou a forte dependência dos resultados em relação à solução arquitetônica utilizada para fechamento da envoltória, de modo que é fundamental que a definição das vedações, principalmente as translúcidas e de sua proteção em relação à radiação solar, seja criteriosamente estudada pelo arquiteto. $\mathrm{O}$ presente estudo mostra que as estratégias adotadas no projeto do Palácio Gustavo Capanema, concebido entre a década de 30 e 40, seguindo estratégias bioclimáticas, e quando ainda não existia uma tecnologia de vidros e de películas de alta eficiência, obtiveram resultados significativamente eficazes.

As avaliações da potência elétrica, do investimento inicial, do consumo elétrico e do custo da energia mostraram que os ganhos obtidos na carga térmica da edificação, necessariamente não são replicados, nas mesmas proporções, para os itens citados. A variação destes itens apresentou forte correlação com a capacidade do equipamento de HVAC instalado, em função das modulações comerciais destes equipamentos. O custo da energia apresentou uma variação de $+21,3 \%$ para a simulação T1 (sem brise e persianas) e $-8,3 \%$ para a simulação T9 (com brise, persianas e vidro de alta eficiência), em relação à simulação $\mathrm{T} 5$ (com brise, persiana e vidro simples). Esta variação foi menor do que a variação do consumo, que foi de $+31 \%$ para a simulação $\mathrm{T} 1$ (sem brise e persianas) e de $-23 \%$ para a simulação T9 (com brise, persianas e vidro de alta eficiência). Apesar de cada projeto possuir características específicas, o presente estudo evidencia a importância do desenvolvimento do projeto de forma integrada, uma vez que se verifica que as decisões de cada disciplina, principalmente as arquitetônicas, impactam diretamente nas demais disciplinas e consequentemente no consumo de energia da edificação.

\section{REFERÊNCIAS}

ASHRAE STANDARD. Standard Method of Test for the Evaluation of building Energy Analysis Computer Programs . ASHRAE 140-2011.

BOECKER J., HORST S., KEITER T., LAU A. e SHEFFER M., TOEVS B. and REED B. The Integrative Design Guide to Green Building - Redefining the practice of Sustainability. New Jersey: John Wiley and Sons, Inc. 2009.

EDWARDS, B. Guía básica de la sostenibilidad, $2^{\circ}$ edição revisada e ampliada. Barcelona: Gustavo Gili, SL, 2008.

IPHAN - Termo de Referência para prestação de serviços de Elaboração Estudos para a Sustentabilidade e Certificação de Edifícios Verdes do Palácio Gustavo Capanema (PGC) - R.J., 2012.

MENDES, N.; WESTPHAL, F.S.; LAMBERTS R.e NETO, J.A.B.C. Uso de instrumentos Computacionais para análise do desempenho térmico e energético de edificações no Brasil. Ambiente Construído, Porto Alegre, out./dez. 2005.

MINDLIN, H.E. - Arquitetura moderna no Brasil - $2^{\mathrm{a}}$ edição. Rio de Janeiro, Aeroplano Editora: IPHAN, 2000.

SILVA, S.R.B. Decisões Arquitetônicas x Potência Instalada para o Sistema de Condicionamento de Ar: Estudo de Caso Palácio Gustavo Capanema - Dissertação de mestrado em Ambiente Construído e Patrimônio Sustentável - Escola de Arquitetura da UFMG, Belo Horizonte, 2014.

TRACE 700 - Comprehensive Building Analysis - Getting Started and Building Energy and Economics Analysis - User's Manual Version 6.2- 2010 . 